\title{
Erratum: Non-Markovian quantum thermodynamics: Laws and fluctuation theorems [Phys. Rev. B 98, 085415 (2018)]
}

\author{
Robert S. Whitney (1) \\ (Received 14 March 2021; revised 29 April 2021; published 13 May 2021)
}

DOI: 10.1103/PhysRevB.103.199901

Section VIII D of the paper contained an error in the condition for the validity of the Crooks equation in the paper's Eq. (67). The Crooks equation still holds in the form given in Eq. (67) but only under less general conditions than were claimed. The correct statement is that the Crooks equation given in the paper's Eq. (67) holds under the condition that the final state in setup $B$ coincides with the time reverse of the initial state in setup A, i.e., $\rho_{\mathrm{sys}}^{(\mathrm{f} 2)}=\rho_{\mathrm{sys}}^{(\mathrm{i})}$. This should replace the erroneous condition that directly followed the paper's Eq. (67). Happily, the applications of the Crooks equation in the paper occurred under the condition $\rho_{\text {sys }}^{(\mathrm{f} 2)}=\rho_{\text {sys }}^{(\mathrm{i})}$ for which the Crooks equation is valid, so the main results and conclusions of the paper are correct.

The error is limited to places in Sec. VIII D where it was claimed that the Crooks equation would hold while $\rho_{\text {sys }}^{(\mathrm{f} 2)} \neq \rho_{\text {sys. }}^{(\mathrm{i})}$. Specifically, the paragraph that starts "One can easily generalize the scenario..." was wrong and should be ignored. In contrast, the example given in Fig. 6 has $\rho_{\text {sys }}^{(\mathrm{f} 2)}=\rho_{\text {sys }}^{(\mathrm{i})}$, so it is entirely correct. Similarly, Sec. IX C's derivation of the steady-state fluctuation relation uses the Crooks equation with $\rho_{\text {sys }}^{(\mathrm{f} 2)}=\rho_{\text {sys }}^{(\mathrm{i})}$, so it is also correct.

Finding the correct condition for the validity of the Crooks equation. The derivation of the Crooks equation in Sec. VIII D required two conditions to be true. One condition was that $\rho_{\text {sys }}^{(\mathrm{f} 2)}$ and $\rho_{\text {sys }}^{(\mathrm{i})}$ were diagonal in the same basis-see below the paper's Eq. (69). The other condition was that

$$
\overline{\Delta S_{\mathrm{tot}}}(\bar{\gamma})=-\Delta S_{\mathrm{tot}}(\gamma)
$$

held for every trajectory $\gamma$. This was used in the paper just above the paper's Eq. (69) where it was incorrectly assumed that this condition would always be true. This is not the case. Instead, one has $\Delta S_{\text {tot }}(\gamma)=\Delta S_{\text {sys }}(\gamma)+\sum_{\alpha} \Delta S_{\text {res }}^{(\alpha)}(\gamma)$ with these entropies defined in the paper's Sec. V. From this one can see that $\overline{\Delta S_{\text {res }}^{(\alpha)}}(\bar{\gamma})=-\Delta S_{\text {res }}^{(\alpha)}(\gamma)$ for all $\gamma$, so the condition in Eq. (i) reduces to the condition that

$$
\overline{\Delta S_{\mathrm{sys}}}(\bar{\gamma})=-\Delta S_{\mathrm{sys}}(\gamma)
$$

holds for all $\gamma$. Here $\Delta S_{\text {sys }}(\gamma)$ is given by the paper's Eq. (20). The only way to satisfy this condition along with the first condition mentioned above (that $\rho_{\text {sys }}^{(\mathrm{f} 2)}$ and $\rho_{\text {sys }}^{(\mathrm{i})}$ are diagonal in the same basis) is to have

$$
\rho_{\text {sys }}^{(\mathrm{f} 2)}=\rho_{\text {sys }}^{(\mathrm{i})} \text {. }
$$

If this is true, all other steps in the derivation follow through as in the paper, but the result is that the Crooks equation in the paper's Eq. (67) only holds under the condition that $\rho_{\text {sys }}^{(\mathrm{f} 2)}=\rho_{\text {sys }}^{(\mathrm{i})}$. Hence, the Crooks equation holds for the scenarios in the paper's Fig. 6 and in the paper's Sec. IX C but does not hold for the scenarios discussed in the paragraph in Sec. VIII D that starts "One can easily generalize the scenario...". Thus, that paragraph should be ignored. 